\title{
Non-hermitian dynamics in delay coupled semiconductor lasers
}

\author{
Andrew Wilkey, Joseph S. Suelzer*, Yogesh Joglekar, Gautam Vemuri \\ Dept. of Physics, Indiana University Purdue University Indianapolis (IUPUI), IN USA 46202-3273 \\ *Air Force Research Laboratory, 2241 Avionics Circle, Wright-Patterson Air Force Base, OH 45433
}

\begin{abstract}
This paper describes our work on the realization of a non-hermitian Hamiltonian system in time-delay coupled semiconductor lasers consisting of two identical lasers, operated with a small frequency detuning between them, and bidirectionally coupled to each other through optical injection. The effective Hamiltonian for this system is non-hermitian, and, under some assumptions and conditions, reminiscent of two-site paritytime (PT) symmetric Hamiltonians, a topic that is under intense investigation. The dynamical response of the intensity of the lasers as a function of the detuning between them reveals characteristics of a PT symmetric system, and our emphasis is on the features that arise from the delayed coupling. Experimental measurements are in good agreement with numerical simulation of the nonlinear rate equation model that describes the coupled system.
\end{abstract}

Keywords: Time-delay coupling, non-hermitian, parity-time symmetry

\section{INTRODUCTION}

Non-hermitian Hamiltonians, and the varying dynamics that result in systems that are described by such Hamiltonians, have attracted much attention in recent years. Part of this interest is driven by the fundamental physics inherent in such systems, and part of it by the putative applications of such systems. It is axiomatic in quantum mechanics that the Hamiltonian is hermitian which then leads to real eigenvalues, orthonormal eigenfunctions, and unitary time evolution, and these Hamiltonias arise in closed systems that are decoupled from the environment. Non-hermitian Hamiltonians arise in open systems wherein coupling to the environment leads to dissipation. One class of non-hermitian Hamiltonians are those that are simultaneously parity $(\mathrm{P})$ and time-reversal $(\mathrm{T})$ symmetric. The pioneering work of Bender and co-workers, ${ }^{1-3}$ and others, ${ }^{4-6}$ demonstrating that such non-hermitian Hamiltonians may have a real energy spectrum provided has led to tremendous interest in experimental realizations of PT-symmetric laboratory systems. ${ }^{7-14}$ The P operator changes the sign of position $x \rightarrow-x$ and momentum $p \rightarrow-p$ and the T operator changes sign of momentum $p \rightarrow-p$ and the sign of complex $i \rightarrow-i$. Some of the early experiments were on coupled pendula ${ }^{13}$, and electrical circuits, ${ }^{14}$ but a majority of the experiments have been in the optical domain. This is largely due to the fact that PT-symmetry can be realized in systems with balanced gain and loss, and since gain and loss are ubiquitous in optics, much effort has been put into developing integrated structures with appropriate gain and loss properties. ${ }^{15}$ The prototypical PT-symmetric dimer consists of two coupled oscillators wherein the gain in one oscillator is exactly equal to the loss in the other. The resulting $2 \times 2$ Hamiltonian matrix which describes this system then has complex diagonal elements, which are complex conjugates of each other and represent gain and loss in each oscillator, and the off-diagonal elements are real and equal and represent the coupling between the oscillators. In recent years, there have been reports on non-hermitian systems in which the potential term in the Hamiltonian is time-dependent, and several papers have reported on such systems that are studied using Floquet methods. ${ }^{16}$

Independently, the nonlinear dynamics of coupled semiconductor lasers (SCLs) has received much attention over the years due to applications that range from linewidth narrowing, and optical waveform generation, to chaotic synchronization. ${ }^{17-24}$ There is a considerable body of knowledge and understanding on how these coupled lasers behave. Coupled lasers are especially attractive for the experimental realization of 
PT-symmetric models and exceptional point (EP) behaviors, and a few recent experiments have fabricated synthetic mircocavity lasers on an integrated chip, and reported the PT-symmetric properties of the system. ${ }^{7}$ The laser configuration is typically designed to exploit the balance between the gain and loss of the laser in order to elicit novel and unexpected behaviors that arise when the system undergoes an abrupt PT phase transition or more generally approaches an exceptional point (EP).

We now report a laboratory realization of a time-delayed, non-hermitian, system in a bulk optical configuration that is comprised of two optically coupled semiconductor lasers (SCLs), and an experimental and theoretical investigation of the properties of this system with special attention to the novel features that arise from the time delayed coupling between oscillators. We show that the rate equation model that is typically used to describe these coupled lasers, can, under certain conditions, lead to an effective nonhermitian Hamiltonian that is strongly reminiscent of the Hamiltonians that arise in the study of conventional PT-symmetric systems. Our experiments demonstrate not only that the coupled SCL system possesses many of the features that PT-symmetric systems do, but also reveals key signatures associated with the time-delayed coupling. Time-delayed differential equations are generally not amenable to analytic solutions and hence we resort to numerical methods to solve the relevant equations that model our system. The predictions of the numerical modeling are compared to the experiments, and the results of are in very good agreement. It is anticipated that the outcomes of our work will be important for systems described by non-Hermitian rate equations, local and nonlocal, and their laboratory implementations.

The most important and distinguishing features of our proposed system are that unlike most nonhermitian systems, which rely on coupling an oscillator with gain to an identical one with loss, our system couples two oscillators in which the frequency detuning between the two lasers, and the coupling strength between them, respectively, are the relevant parameters. The advantages of this are

(i) In contrast to other systems where a precise balance between gain and loss has be engineered, our system automatically ensures that the frequency detuning of one laser is exactly equal and opposite in sign to the frequency detuning of the second laser thereby guaranteeing that the diagonal elements of the PT-Hamiltonian are equal and opposite in sign.

(ii) The physical separation between the two lasers naturally introduces a time-delay in the coupling, and hence permits a study of time-delay effects in non-hermitian systems. The time-delayed aspect is a novel and unexplored topic in the field of PT-symmetry.

PT-symmetric systems are also of interest for their putative applications ${ }^{25-37}$ such as making materials with unidirectional optical propagation, ${ }^{36}$ single mode lasing action, ${ }^{32}$ and the spontaneous generation of photons in a PT-symmetric medium by a vacuum field. ${ }^{37}$ Due to the miniature size of SCLs, and well established fabrication methods for incorporating several lasers and associated components on chips, our work may lead to PT-symmetric photonics on a chip.

\section{NUMERICAL MODEL}

Our system is described by a rate equation model that is based on the well-known Lang-Kobayashi model ${ }^{38}$ wherein we assume that the two lasers are identical and operate at frequencies, $\omega_{1}$ and $\omega_{2}$. The slowly varying electric fields inside the two laser cavities are defined in a symmetric reference frame that is given by the average of the two optical frequencies, i.e. $\theta=\frac{1}{2}\left(\omega_{1}+\omega_{2}\right)$. The rate equations describing the normalized complex electric fields $E_{1,2}$ and the normalized excess carrier densities $N_{1,2}$ may be written as follows ${ }^{39}$ : 


$$
\begin{aligned}
& \frac{d E_{1}}{d t}=(1+i \alpha) N_{1}(t) E_{1}(t)-i \Delta \omega E_{1}(t)+\kappa e^{-i \theta \tau} E_{2}(t-\tau) \\
& \frac{d E_{2}}{d t}=(1+i \alpha) N_{2}(t) E_{2}(t)+i \Delta \omega E_{2}(t)+\kappa e^{-i \theta \tau} E_{1}(t-\tau) \\
& \mathrm{T} \frac{d N_{1}}{d t}=J_{1}-N_{1}(t)-\left(1+2 N_{1}(t)\right)\left|E_{1}(t)\right|^{2} \\
& \mathrm{~T} \frac{d N_{2}}{d t}=J_{2}-N_{2}(t)-\left(1+2 N_{2}(t)\right)\left|E_{2}(t)\right|^{2}
\end{aligned}
$$

where $\alpha$ is the linewidth enhancement factor, ${ }^{29} \tau$ is the time delay in coupling, $J_{1,2} \propto I_{1,2} / I_{t h r}$, is the normalized pumping, $I_{t h r}$ is the injection current above threshold and $T=\tau_{s / \tau_{p}}$ is the ratio of the carrier lifetime to the photon lifetime. The important and relevant parameters are $\kappa$ and $\Delta \omega=\left(\omega_{1}-\omega_{2}\right)$ which describe the coupling coefficient and the frequency detuning respectively. The above model has been used with great success to describe the nonlinear dynamical behavior of coupled SCLs in a number of different papers. Note that in Eq. 1-4 above, the coupling term has been modified to account for the mutual coupling between the two lasers and a phase shift term has been added that depends linearly on the frequency detuning, $\Delta \omega$. The linear dependence implies that higher order coupling terms have been neglected, an approximation that is known to work well for weak coupling strengths of less than $5 \%$ of the optical power of the lasers. We note from Eq. 1-4 that it is already apparent that $\pm i \Delta \omega$ play the role of gain/loss in conventional PT systems. In our system, $\pm i \Delta \omega$ accounts for frequency pulling that is typical of coupled lasers operating at slightly different frequencies.

To motivate the connection to non-hermitian Hamiltonians in general, and PT-symmetry in particular, and to gain some insight into the rate equations, first consider the case of zero delay $(\tau=0)$. When the SCLs are operating in steady state, above threshold, the inversion above transparency is zero, i.e $N_{1,2} \sim 0 .{ }^{40}$ Therefore, Eq. 1-4 reduces to

$$
\left(\begin{array}{l}
\dot{E}_{1}(t) \\
\dot{E}_{2}(t)
\end{array}\right)=\left(\begin{array}{cc}
i \Delta \omega & \kappa \\
\kappa & -i \Delta \omega
\end{array}\right)\left(\begin{array}{l}
E_{1}(t) \\
E_{2}(t)
\end{array}\right)
$$

where the 2x2 effective Hamiltonian is clearly non-Hermitian and similar to typical PT-symmetric Hamiltonians, with the difference being that the diagonal elements of the matrix that normally represent gain/loss terms are replaced in Eq. (5) by frequency detuning between the two lasers. There are other important distinctions between our model and typical PT-systems. First, the SCL model is a rate equation model in contrast to typical PT-systems that are studied by invoking the Schroedinger equation. Thus, the complex $i$ that occurs in the Schroedinger equation is missing in our model, and leads to important differences. ${ }^{41}$ For example, the diagonal elements, instead of contributing to amplification or attenuation of light, now give rise to temporal oscillations in the field. Second, the off-diagonal elements, instead of determining the frequency of exchange between the two oscillators, now contribute to laser intensity growth.

We note that despite the simplifying assumptions made above to get Eq. (5), and the differences in the conventional PT model and our system, the coupled SCL system does behave like a PT-symmetric system, as we will show in the results sections. In fact, our work to date indicates that the coupled SCL system is a very robust PT system, and that the signatures of PT symmetry remain even without the simplifying assumptions. Lastly, our experiments also show a similar robustness.

\section{EXPERIMENTS}

Our experiments are based on variations of the system in Fig. 1 that was used to obtain the results described in previous work ${ }^{30}$ and is shown schematically below. It consists of two single mode (HL7851G) 
semiconductor lasers (SCL1, SCL2), an external cavity consisting of two beam splitters (BS1 \& BS2) which optically couple the two SCLs, and an external control of the coupling strength, $\kappa$, via a variable neutral density filter (VND). The SCLs are identical except for their free running optical frequencies and threshold pump currents. The transmission through the VND is determined by an independent laser and photodiode which allows us to calibrate the experimental and theoretical coupling strength, $\kappa=\left(1-r^{2}\right) /\left(r \tau_{i n}\right) \xi \tau_{p}$, where $r$ is the reflectivity of the external laser facet, $\xi^{2}$ is the fraction of optical power transmitted by all the optical elements, $\tau_{i n}$ is the internal laser round trip time and $\tau_{p}$ is the photon lifetime. Once the transmission through the VND is recorded, $\xi^{2}$ can be determined since all the other optical elements are fixed. This model assumes that the fractional power is fully coupled into the active region of the SCL. However, due to the relative sizes of the beam profile $(>100 \mu \mathrm{m})$ and the active region (about $10 \mu \mathrm{m}$ ), only a portion of the power is coupled into the active region. Through literature reports, and our comparisons of theory to experiment, we find that after determining $\xi^{2}$, we must divide it by 10 to use in simulations. The experiment is designed such that the coupling strengths to the two lasers are equal, and a Faraday rotator is placed in the coupling beam path to eliminate self coupling. The glass slides (GS1 \& GS2) independently reflect a portion of (8\%) the intensity from SCL1 and SCL2, and the measurement of the laser intensities is done using two $1 \mathrm{GHz}$ photodiodes in conjunction with a $1 \mathrm{GHz}$ oscilloscope. The currents and temperatures of the lasers are stabilized to $0.01 \mathrm{~mA}$ and $0.01 \mathrm{C}$ respectively.

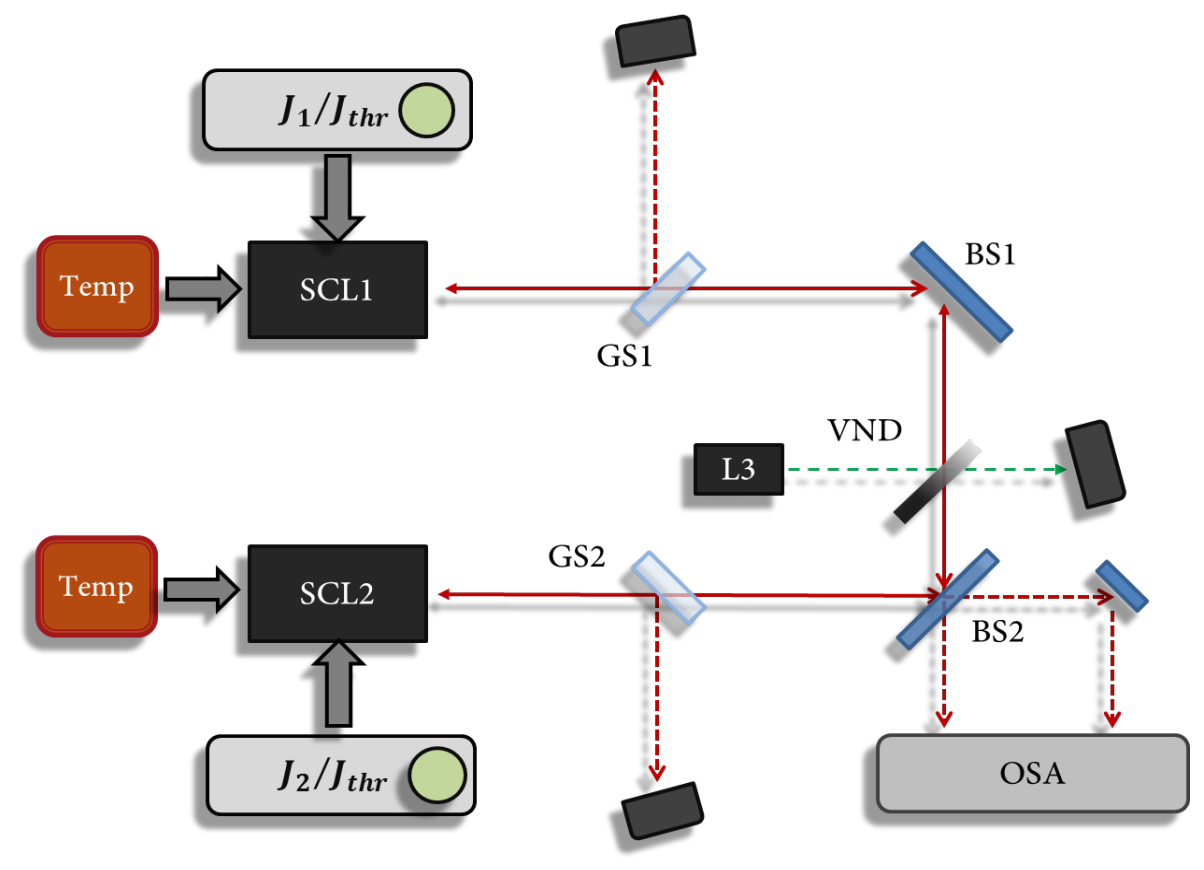

Fig. 1. Experimental schematic. SCL: semiconductor laser, GS: glass slide, VND: variable neutral density filter, OSA: optical spectrum analyzer, L3: laser used to quantify coupling strength, BS; beam splitter.

After bidirectional coupling of the lasers, the temperature to SCL1 is scanned at a slow rate $(<10 \mathrm{~Hz})$ and the intensities of the two lasers monitored. The important parameters, $\kappa$ and $\Delta \omega$, can be varied via the VND and the temperature of SCL1, respectively. It is important to point out that for unequal coupling strengths, or for unidirectional coupling, one gets very different behaviors from a PT-system. For uncoupled 
lasers, the intensity of SCL2 remains constant and that of SCL1 decreases in proportion to the temperature increase. For unidirectional coupling, the intensity of the slave laser follows an intensity profile identical to an injection laser.

We next discuss how we vary $\Delta \omega$. For our lasers near room temperature, the optical emission frequency is proportional to the temperature of the active region. Since the experimental procedure requires that we change the temperature by less than $4 \mathrm{C}$, it is safe to assume a linear dependence of the emission frequency on the temperature. The temperature dependence of the optical frequency and intensity can be approximated by

$$
\begin{aligned}
& \omega(T)=\omega_{o}-k_{T} T \\
& I(T)=I_{t h r}+\eta_{T} T
\end{aligned}
$$

where $k_{T}=20 \mathrm{GHz} / \mathrm{C}$ and $\eta_{T}=0.15 \mathrm{~mW} / \mathrm{C} . k_{T}$ is experimentally determined by scanning the temperature of one SCL while monitoring the intensity through a fixed $2 \mathrm{GHz}$ free spectral range Fabry-Perot etalon. When $n$ number of peaks are observed through the etalon, the proportionality constant can be obtained from

$k_{T}=(2 \mathrm{GHz}) n / \Delta T$ where $\Delta T$ is the range of temperature scanned. To obtain $\eta_{T}$, the scanned temperature and the emitted laser light are recorded. The linear relationship can be directly determined from this measurement, $\Delta I / \Delta T$.

Above PT-threshold $(\mid \Delta \omega>\kappa)$, the simplified PT-model and simulation of the full laser model show that the inclusion of time-delay causes oscillations in intensity as a function of $\Delta \omega$, and these oscillations are a result of oscillations in the sign of the real part of the eigenvalues. To test this, our typical experiment operates two SCLs at constant injection current about 3\% above their respective lasing thresholds. This guarantees the SCL to remain above threshold when the temperature is scanned. The injection current is tuned to a precision of $0.01 \mathrm{~mA}$ to ensure that the output power of each laser is identical. The optical spectrum of the uncoupled (free-running) SCLs are independently measured by an HP 7554 scanning optical spectrum analyzer. The temperature to one laser is scanned and recorded. Using Eq. (6) along with temperature and wavelength measurements, we calculate the detuning, $\Delta \omega$. The temperature, coupling strength and the two SCL intensities are simultaneously recorded resulting in an intensity profile. The photodiodes are operated with a large load resistor to decrease the bandwidth to $<1 \mathrm{GHz}$. This bandwidth, along with the scan rate of the oscilloscope, time average the intensities.

\section{RESULTS}

The eigenvalues of the matrix in Eq. (5) are real (complex) when $\kappa>\mid \Delta \omega(\kappa<\mid \Delta \omega)$. Therefore, one expects an abrupt increase in the intensities of the lasers when $\kappa$ is swept across the threshold, i.e. the PTtransition should occur at $\kappa=\mid \Delta \omega$. We have confirmed this through numerical simulations of the full laser model, as given by Eq. (1-4), as well as through experiments. In what follows, we show some representative results in which all time scales are in units of the photon lifetime. For comparison to real time scales, note that the photon lifetime is typically about $10 \mathrm{ps}$, and so a $\kappa$ of 0.1 in scaled units would be $10 \mathrm{GHz}$, and a time delay, $\tau$, of 100 would be 1 ns.

In Fig. $2 \mathrm{a}$ are shown the real and imaginary parts of the eigenvalues obtained from the simplified $2 \times 2$ model of Eq. (5) as a function of the detuning frequency, for a $\kappa$ of 0.1 , and zero time delay. 

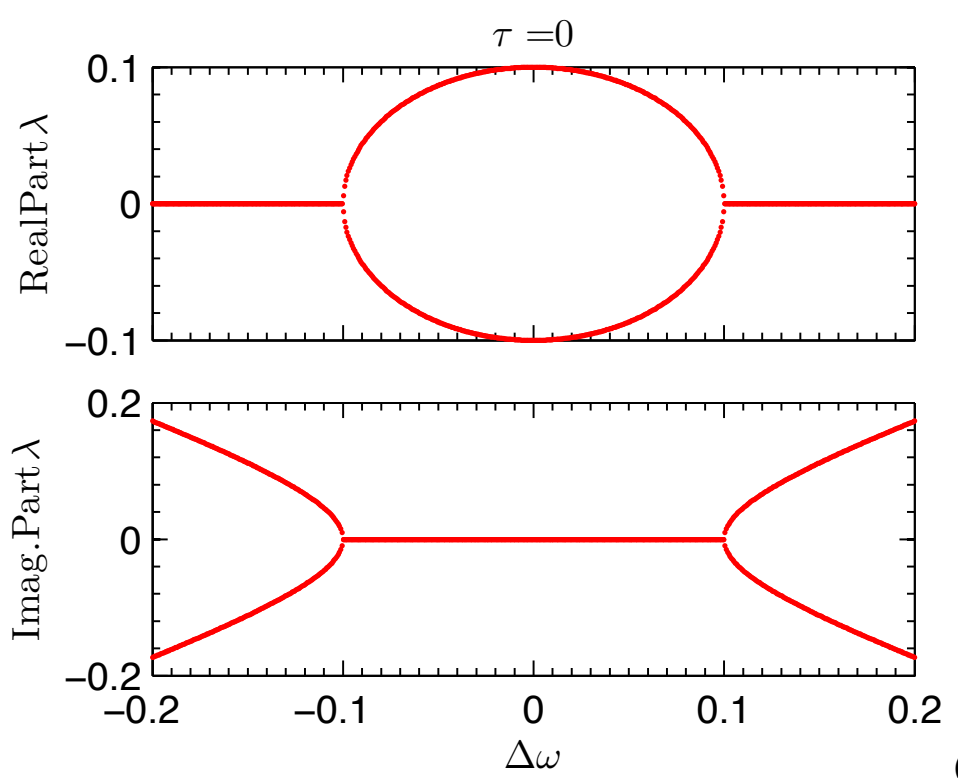

(a)

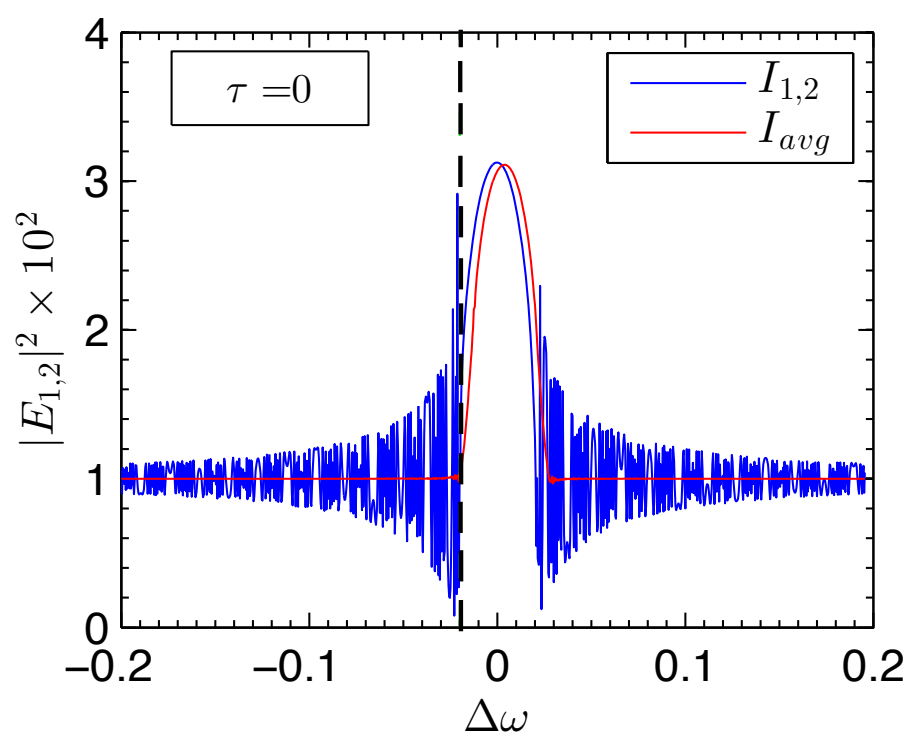

(b)

Fig. 2 (a) Trajectories of the eigenvalues when $\kappa=0.1$ and $\tau=0$. (b) Simulations of the SCL intensities when $\kappa=0.02$ for zero delay. Solid blue line is instantaneous intensities of both lasers, red line is time-averaged intensity over detector risetime of $10 \mathrm{~ns}$, and vertical dashed black line is predicted PT-transition point where $\kappa=\Delta \omega$.

Clearly, for $\kappa<|\Delta \omega|$ the eigenvalues are entirely imaginary leading to oscillations in the intensity of the laser, whereas for $\kappa>|\Delta \omega|$ the eigenvalues are real and lead to growth or decay of laser intensity. In Fig. $2 \mathrm{~b}$ are the intensities of the two lasers obtained from the full laser model of Eq. (1-4) with $\kappa$ of 0.02 and zero time delay. The solid blue line shows the intensities of the two lasers that are predicted in this regime, and the red line is a rolling time average with a detector bandwidth of $100 \mathrm{MHz}$, so that we mimic the experimental conditions. Note that the observations of intensity oscillations for $\kappa<|\Delta \omega|$ and intensity growth for $\kappa>$ $|\Delta \omega|$ are reproduced exactly as predicted for the simplified $2 \times 2$ matrix model. This is a significant result since it demonstrates that the full laser model retains the characteristics of a PT-symmetric system. It is this 
robustness of the system, coupled with the simplicity of its implementation, that makes it a very attractive candidate for studying non-hermitian dynamics.

A novel feature of our system, and a major focus of our work, is the time-delay in the SCL model. The introduction of a time-delay has important methodological consequences - (i) we note from Eq. (1-4) that one cannot ignore the phase accumulation term, viz. $e^{-i \theta \tau}$ and (ii) one now has a delay differential equation, the solution of which requires suitable techniques. Analytically, one seeks solutions of the form $e^{\lambda \tau}$, and expresses the delayed terms in Eq. (1) and (2) as $E(t-\tau) \propto E(t) e^{-\lambda \tau}$. From Eq. (1) and (2), we see that the coupling term now becomes $\kappa e^{-i \theta \tau} e^{-\lambda \tau} E(t)$. The effective, semiclassical Hamiltonian now has the form

$$
\left(\begin{array}{cc}
i \Delta \omega & \kappa e^{-\lambda \tau} \\
\kappa e^{-\lambda \tau} & -i \Delta \omega
\end{array}\right)
$$

As expected, the effects of the delay appear in the off-diagonal elements, $\kappa$. Furthermore, it is clear that if the eigenvalues (i.e. $\lambda$ ) are complex, then this effective Hamiltonian may no longer be PT-symmetric. To examine this, we show in Fig. 3 a the eigenvalues vs. $\Delta \omega$ for a $\kappa$ of 0.1 and time delay of 100 units (in normalized units, corresponds to a real delay of $1 \mathrm{~ns}$ ) where we find that the system still retains the traits of a PT-symmetric system. There is a distinct transition from real to imaginary eigenvalues at $\kappa=|\Delta \omega|$, where the imaginary component of the eigenvalue becomes zero and the real part becomes finite. The most interesting features occur when $|\Delta \omega|>\kappa$, at which point the real part of the eigenvalues oscillates from positive to negative, while the imaginary part is similar to the zero delay case of Fig. 2a, except for a series of discrete steps. In Fig. $3 b$ are the simulations of the full SCL model given by Eq. (1-4), for $\kappa$ of 0.02 and delay of 100, and remarkably, the intensity oscillations in the $|\Delta \omega|>\kappa$ range are captured by the time-averaged intensities. As shown in Fig. 2b, the time-averaged intensities are constant for zero delay, whereas with a delay the intensities of both lasers oscillate. The vertical, dashed black line marks the position of the expected PT-transition and we see that the simulations are very close to the expected transition.

The most immediate consequence of a time-delayed PT-symmetric system is oscillations in the intensities of the lasers with frequency detuning. A time-delayed PT system gives rise to two types of oscillatory dynamics - (i) the imaginary eigenvalues in the PT broken phase give rise to temporal oscillations, just as in the zero delay case, which occur for fixed frequency detuning and (ii) the time-delay gives rise to oscillations in the real part of the eigenvalues which then manifest themselves as intensity oscillations when the frequency detuning is scanned. The latter can be observed by time-averaging over the (typically) faster temporal oscillations. 

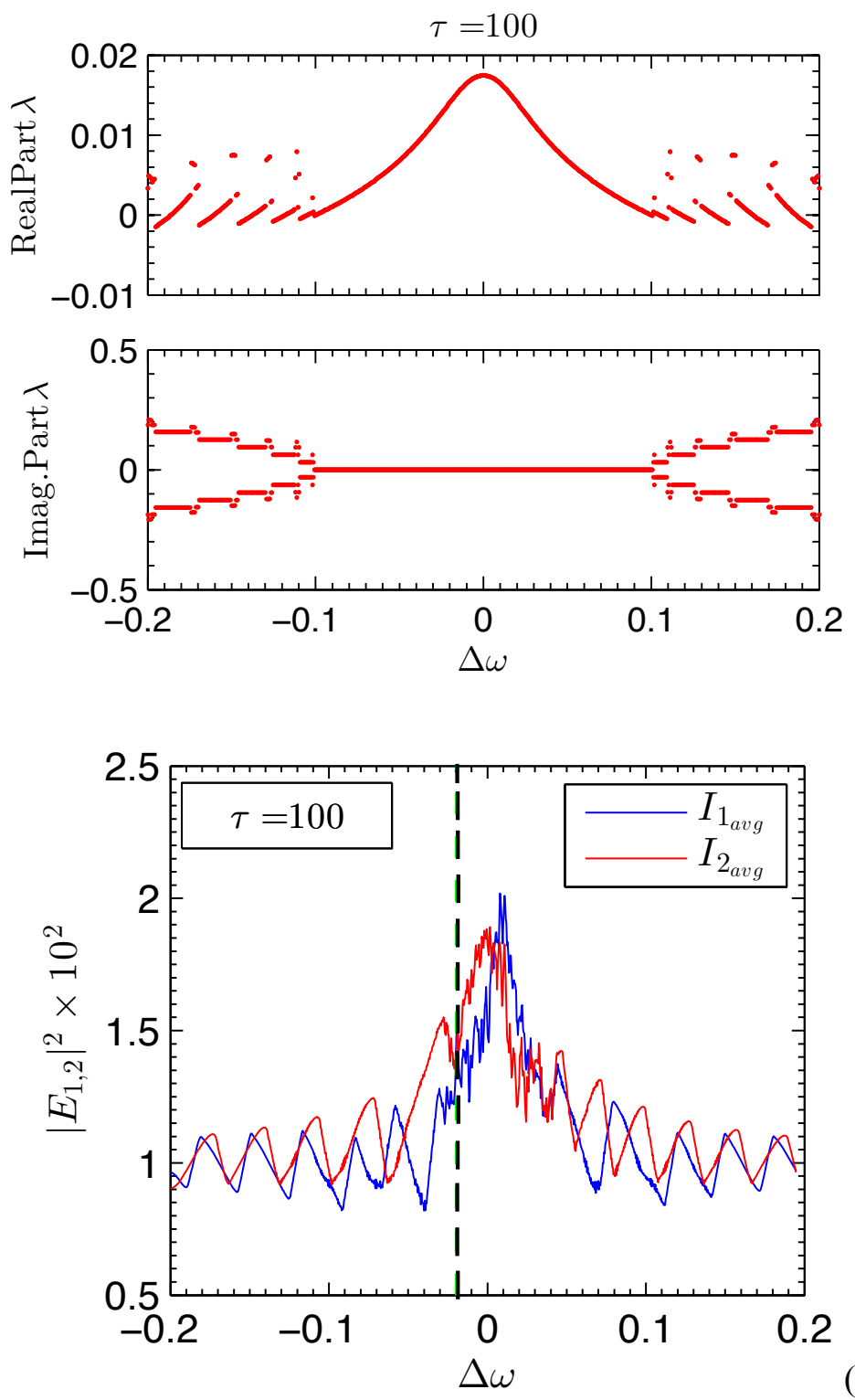

(b)

Fig. 3. (a) Trajectories, at constant phase $(\theta \tau=2 \pi)$, of eigenvalues when $\kappa=0.1$ and $\tau=100$. (b) Simulations of SCL intensities when $\kappa=0.02$ and time delay of 100 . Solid blue $\&$ red lines are intensities of both lasers, time-averaged over $10 \mathrm{~ns}$, and vertical dashed black line is predicted PT-transition point.

Finally, since our experimental procedure relies on scanning the frequency of one laser, i.e. a scan of $\theta$, which is the average of the laser frequencies, and hence a variation in the feedback phase, inclusion of this phase change leads to an effective Hamiltonian of the form

$$
\left(\begin{array}{cc}
i \Delta \omega & \kappa e^{-\lambda \tau} e^{-i \theta \tau} \\
\kappa e^{-\lambda \tau} e^{-i \theta \tau} & -i \Delta \omega
\end{array}\right)
$$

It is important to point out here that this Hamiltonian allows additional control over the non-Hermiticity of the system through a phase control of the off-diagonal element. By varying either $\theta$ or $\tau$, one can, in principle, vary $e^{-i \theta \tau}$ from purely real to purely imaginary. 
The eigenvalues of Eq. (9) are depicted in Fig. 4a, where, once again, we see oscillations in the real part of the eigenvalues and discrete steps in the imaginary ones. A closer look reveals
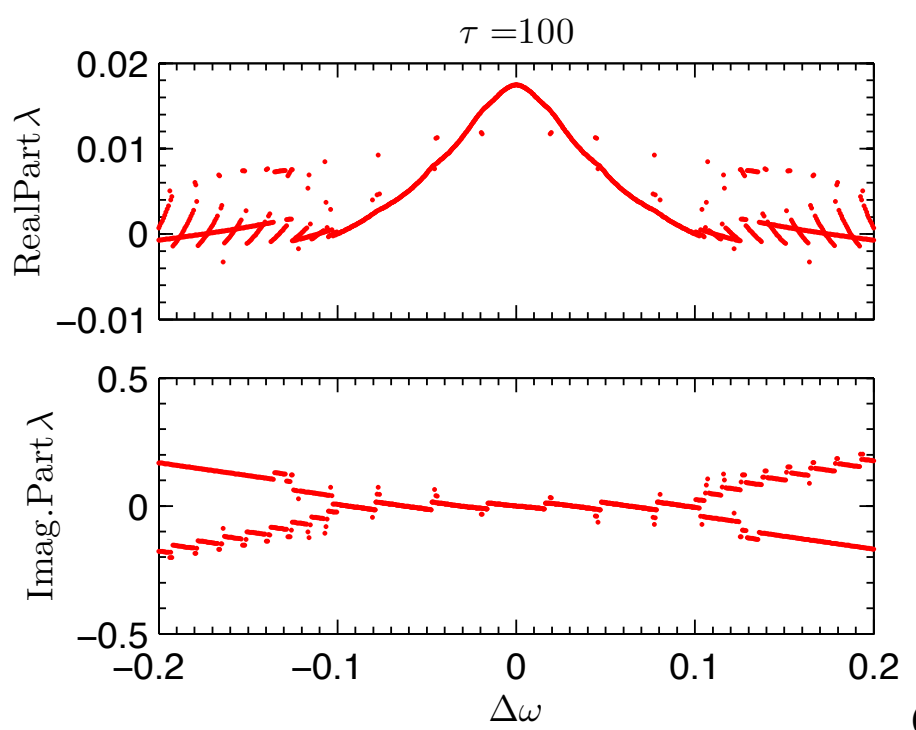

(a)

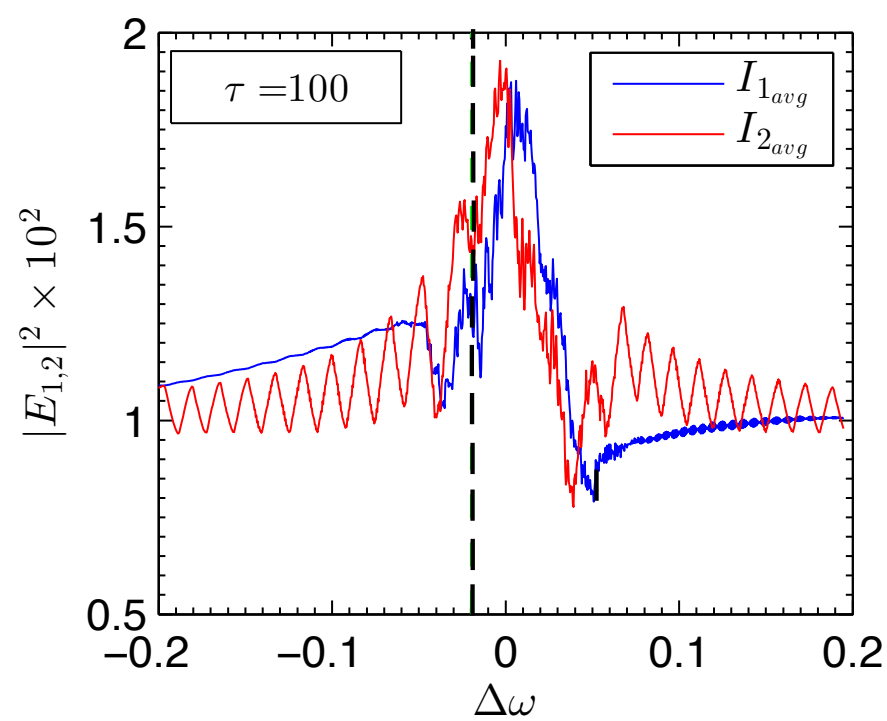

(b)

Fig. 4. (a) Trajectories, with varying phase, of the eigenvalues when $\kappa=0.1$ and $\tau=100$. (b) Simulations of the SCL intensities when $\kappa=0.02$ and time delay of 100. Solid blue \& red lines are instantaneous intensities of both lasers, timeaveraged over $10 \mathrm{~ns}$, and vertical dashed black line is predicted PT-transition point.

that the frequency of the oscillations in the former has increased. In addition, the smooth curve in the real eigenvalues plot between $\Delta \omega=0.12 \rightarrow 0.2$ and $\Delta \omega=-0.2 \rightarrow-0.12$ lays over the discrete jumps unlike in Fig. 3a. It is remarkable that both the features, viz. the increased frequency and the continuous curve are captured in the simulations of the full model. Shown in red line, the intensity of SCL2 undergoes oscillations in the predicted detuning regime whereas the intensity of SCL1 (in blue) is steady (compare to Fig. 3b). 
Next we discuss the experimentally measured laser intensity profiles, obtained with the system of SCL1 and SCL2 for a time delay, $\tau=180$, and $\tau=480$. The temperature of SCL2 (blue) is scanned and the intensities of both SCLs are recorded (Fig. 5a, 5c respectively). We normalize the measured intensities with respect to the minimum recorded intensity to contrast the fractional growth of each SCL intensity. The profiles show an abrupt increase in intensity at $|\Delta \omega|=\kappa$, a clear signature of the PT transition. Especially interesting is that in the region where $|\Delta \omega|>\kappa$, the intensity profiles oscillate, and become more rapid as the time-delay increases, and the amplitude of these oscillations diminish with increasing time-delay.

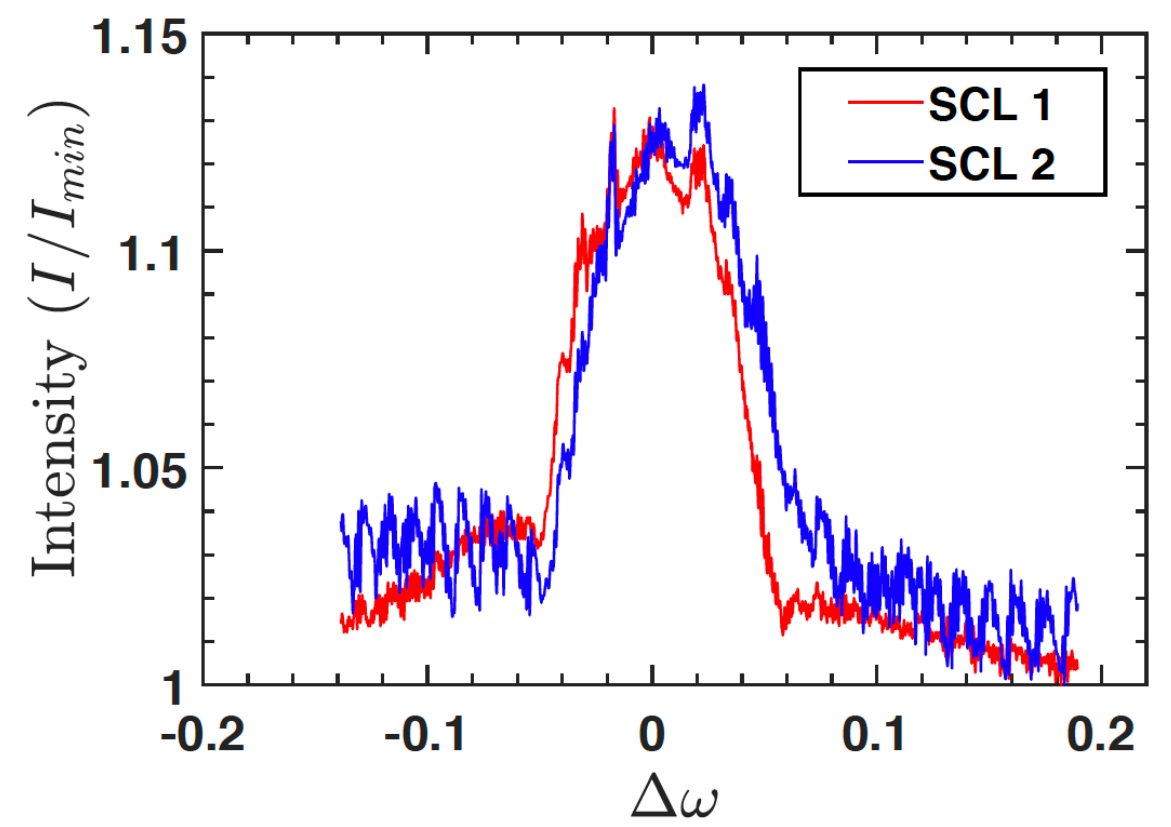

(a)

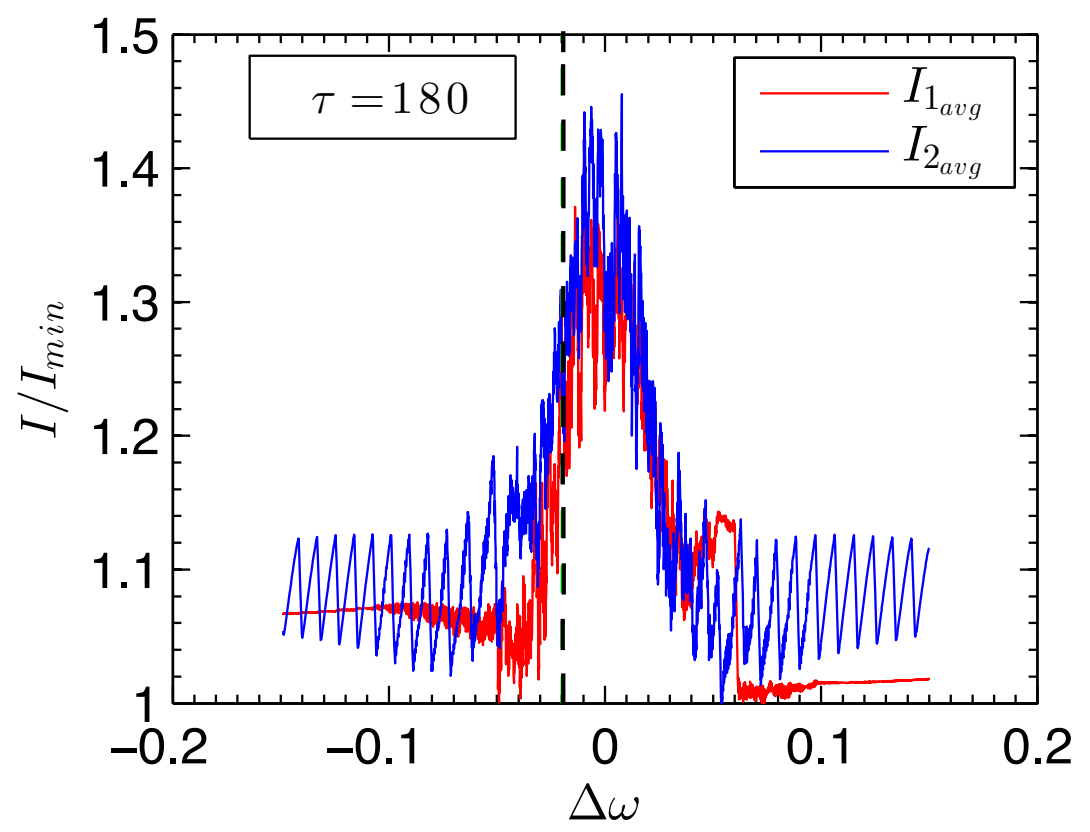

(b) 


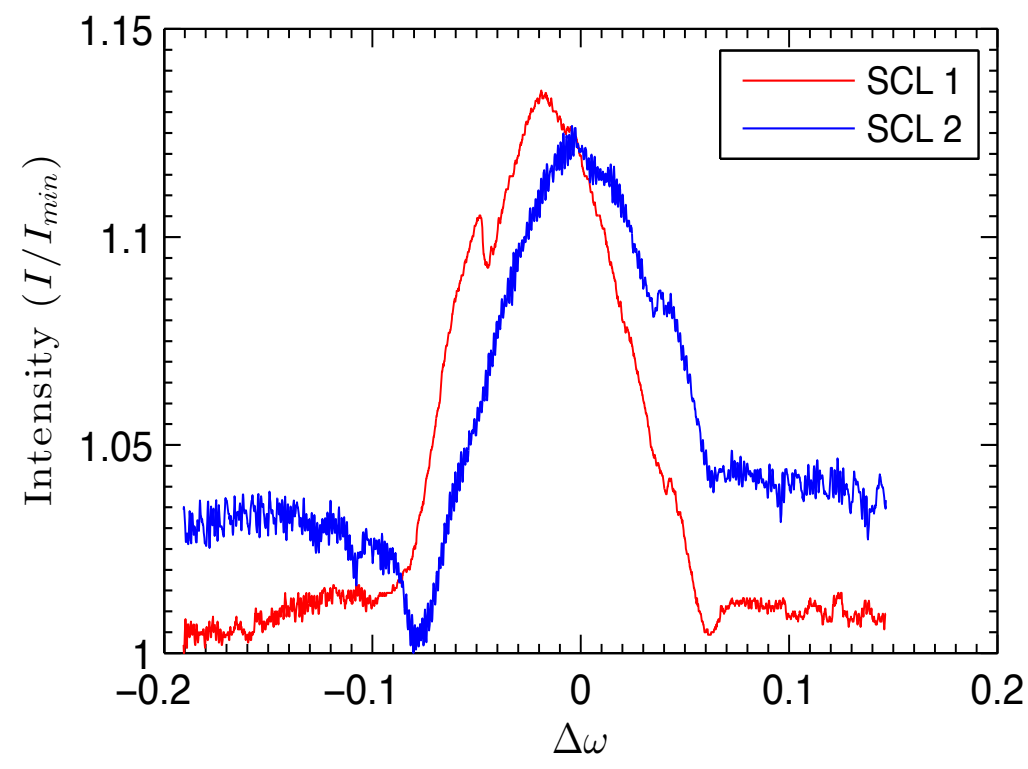

(c)

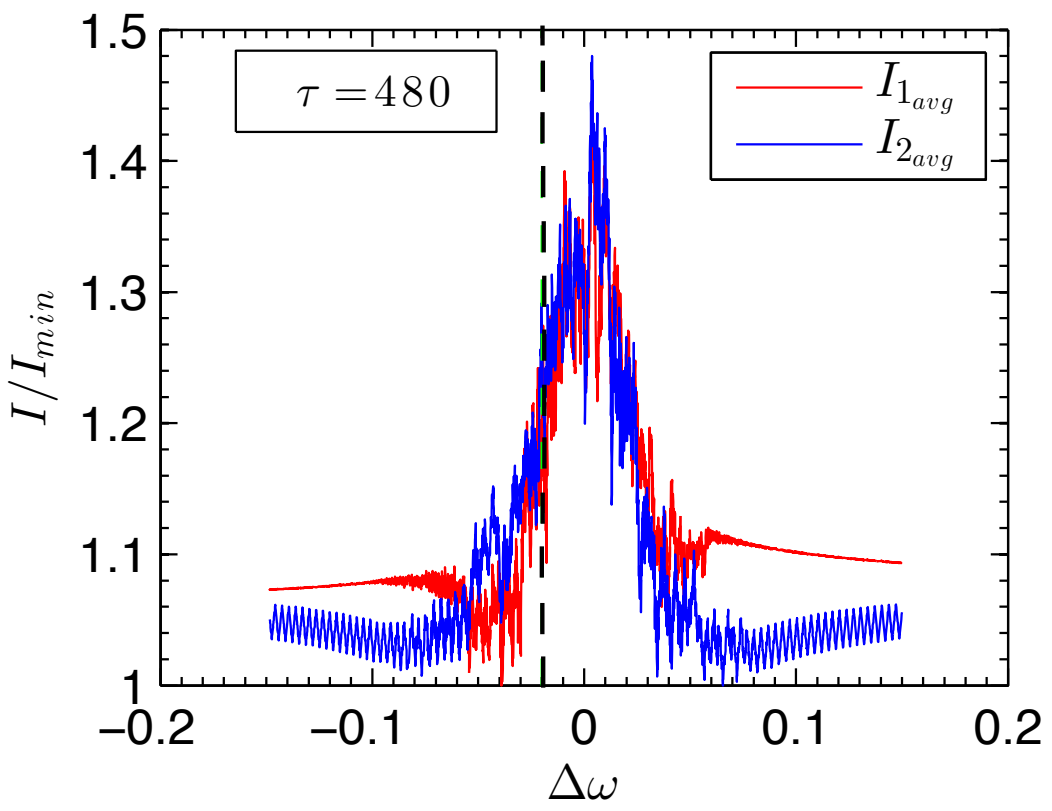

(d)

Fig. 5 (a) Experimental intensity profiles for time delay, $\tau=180$; (b) simulations of the full laser model at, $\tau=180$; (c) experimental intensity profiles for time delay, $\tau=480$; (d) simulations of the full laser model at, $\tau=480$.

These oscillations are a unique characteristic of the time-delayed coupling between the two lasers. For the same parameters as the experiments, the simulation results of the full laser model of Eq. (1-4), are shown in Fig. $5 \mathrm{~b}$ and $5 \mathrm{~d}$, respectively. The numerical results also show that the PT-transition occurs at $|\Delta \omega|=\kappa(=$ 0.02) indicated by the black, dashed vertical line in Fig. 5b, 5d. It is seen in both experiments and simulations that the intensity of SCL2 increases abruptly for a certain detuning between the lasers and this occurs at the PT-threshold. The features noted above were verified up to a $\tau$ of 500 , after which the laser beam divergence 
causes unavoidable problems with coupling and stability of the setup. At the same time, very small delays are also difficult due to the physical footprints of the various optical components.

\section{CONCLUSIONS}

This paper has reported the results of our work on using two, bidirectionally coupled semiconductor lasers to effectively mimic a non-hermitian system and probe its intensity dynamics. The system is entirely classical and yet produces many of the signatures that are common to other PT-symmetric systems. The coupled laser system described here contains many complicating factors, such as nonlinear gain and the resulting saturation effects, time-delay in the coupling between the lasers, and others, and hence it is surprising that the system still retains many of the features of PT symmetry. We have motivated the existence of such behavior through a simplified model which reduces the Lang-Kobayashi model to a $2 \times 2$ matrix which is reminiscent of a PT symmetric dimer. Through this, our work indicates that the detuning between the lasers plays the role of gain and loss in conventional PT symmetric systems. Our experimental measurements and numerical simulations of the Lang Kobayashi model are in qualitative agreement with each other, and both show that many of the features predicted by the simplified model survive in the presence of nonlinearities and gain saturation. We have also shown that the time-delayed coupling has an important effect on the behavior of the system, and that in particular it leads to oscillations in the intensities of one of the lasers as a function of the detuning between the two lasers. Finally, we note that the experiments were done with commercially available lasers, and that all of the important parameters, viz. the detuning between the lasers, the coupling between them, and the time delay, are all easily accessible in experiments.

This work raises many important and interesting questions which will be the subject of future reports. These include the role of quantum noise in these systems, as well the population dynamics at the PT transition. Finally, since the time-delay influences the phase accumulation factor, a detailed study of the phase dynamics of these systems could also be investigated.

\section{REFERENCES}

[1] Bender, C. M. and Boettcher, S. "Real spectra in non-hermitian hamiltonians having PT-symmetry," Phys. Rev. Lett. 80, 5243-5246 (1998).

[2] Bender, C.M., Brody, D. C. and Jones, H.F., "Complex extension of quantum mechanics," Phys. Rev. Lett 89, 270401 (2002).

[3] Bender, C.M., Hook, D.W., Meisinger, P.N. and Wang, Q.-h., "Complex correspondence principle," Phys. Rev. Lett. 104, 061601 (2010).

[4] Scolarici, G. and Solombrino, L., "On the pseudo-hermitian nondiagonalizable hamiltonians," Journal of Mathematical Physics 44(10) (2003).

[5] Brody, D.C., "Consistency of pt-symmetric quantum mechanics," Journal of Physics A: Mathematical and Theoretical 49(10), 10LT03 (2016).

[6] Mostafazadeh, A., "Pseudo-hermiticity versus pt symmetry: The necessary condition for the reality of the spectrum of a non-hermitian hamiltonian," Journal of Mathematical Physics 43(1) (2002).

[7] Rüter, C.E., Makris, K.G., El-Ganainy, R., Christodoulides, D.N., Segev, M., and Kip, D., "Observation of parity-time symmetry in optics," Nature Physics 6(3), 192-195 (2010).

[8] Kottos, T., "Optical physics: Broken symmetry makes light work," Nature Physics 6(3), 166-167 (2010).

[9] Regensburger, A., Bersch, C., Miri, M.A., Onishchukov, G., Christodoulides, D.N., and Peschel, U., "Parity-time synthetic photonic lattices," Nature 488(7410), 167-171 (2012).

[10] Cole, J.T., Makris, K.G., Musslimani, Z.H., Christodoulides, D.N., and Rotter, S., "Twofold PT symmetry in doubly exponential optical lattices," Phys. Rev. A 93, 013803 (Jan 2016). 
[11] Wimmer, M., Regensburger, A., Miri, M.A., Bersch, C., Christodoulides, D.N, and Peschel, U., "Observation of optical solitons in pt-symmetric lattices," Nature communications 6 (2015).

[12] Rivolta, N.X.A., and Maes ,B., "Symmetry recovery for coupled photonic modes with transversal pt symmetry," Opt. Lett. 40, 3922-3925 (Aug 2015).

[13] Bender , C.M., Berntson , B., Parker , D. and Samuel , E., "Obesrvation of PT phase transition in a simple mechanical system", Am. J. of Phys 81(3) (2012).

[14] See e.g. Ramezani, H., Schindler, J., Ellis , F.M., Guenther , U. and Kottos , T., "Bypassing the bandwidth theorem with PT symmetry", Phys. Rev. A85, 062122 (2012).

[15] Zyablovsky , A.A., Vinogradov , A.P., Pukhov , A.A., Dorofeenko , A.V., and Lisyansky , A.A., "Ptsymmetry in optics," Physics-Uspekhi 57(11), 1063 (2014).

[16] Joglekar, Y.N., Marathe , R., Durganandini , P. and Pathak , R.K., "PT spectroscopy of the Rabi problem," Phys. Rev. A90, 040101 (R) (2014).

[17] Soriano, M.C., Garcia-Ojalvo, J., Mirasso , C.R. and Fischer, I., "Complex photonics: Dynamics and applications of delay-coupled semiconductor lasers," Rev. of Mod. Physics 85, 421-470 (2013) and references therein.

[18] Takeuchi , Y., Shogenji , R., and Ohtsubo , J., "Chaotic dynamics in semiconductor lasers subjected to polarization-rotated optical feedback," Applied Physics Letters 93(18) (2008).

[19] Fader , W., “Theory of two coupled lasers," IEEE Journal of Quantum Electronics 21, 1838-1844 (1985).

[19] Sciamanna, M. and Shore, K.A., "Physics and applications of laser diode chaos," Nature Photonics 9, 151-162 (2015)

120] Prasad, A., Ghosh, R. and Vemuri, G., "Effects of Quantum Noise on the Nonlinear Dynamics of a Semiconductor Laser subject to two Spectrally Filtered , Time-Delayed Optical Feedbacks," Optics Commun. 370, 209-221, (2016).

[21] Lang, R., "Injection locking properties of semiconductor lasers," IEEE J. Quantum Electronics 18, 976-983 (1982).

[22] Morgensen, F., Olsen, H. and Jacobsen, G., "Locking conditions and stability properties for a semiconductor laser with external light injection," IEEE J . Quantum Electronics 21, 784-793 (1985). [23] Erzgraeber, H., Lenstra, D., Krauskopf, B., Fischer, A.P.A. and Vemuri, G., "Feedback Phase Sensitivity of a Semiconductor Laser Subject to Filtered Optical Feedback: Experiment and Theory," Phys. Rev. E76, 026212 (2007).

[24] Krauskopf, B. and Lenstra, D. Lenstra, editors [Fundamental issues of nonlinear laser dynamics], AIP Conf Proc., Texel, The Netherlands (2000).

[25] Zhang, X.L., Wang, S., Hou, B. and Chan, C.T., "In Situ Control of Encircling Loops and the Role of the Starting Point," Phys. Rev. A8, 021066 (2018).

[26] Hodaei, H., Hassan, A. U., Wittek, S., Garcia-Gracia1, H., El-Ganainy, R., Christodoulides , D. N., and Khajavikhan , M., "Enhanced Sensitivity at Higher-Order Exceptional Points," Nature 548, 187

(2017).

[27] Chen, W., Özdemir, S. K., Zhao, G., Wiersig, J., and Yang, L., "Exceptional Points Enhance Sensing in an Optical Microcavity,"Nature 548, 192 (2017).

[28] Doppler, J., Mailybaev , A.M., Bohm, J., Kuhl, U., Girschik , A., Libisch , F., Milburn , T.J.,

Rabl, Moiseyev , P., N. and Rotter, S., "Dynamically Encircling an Exceptional Point for Asymmetric Mode Switching," Nature 537, 76-79 (2016).

[29] Choi, Y., Hahn, C., Yoon, J.W. and Song, S.H., "Observation of an anti-PT-symmetric Exceptional

Point and Energy-difference Conserving Dynamics in Electrical Circuit Resonators," Nature Commun. 9, 2182 (2018). 
[30] Suelzer, J., Joglekar , Y. and Vemuri , G., "Parity-Time Symmetry Breaking in Optically Coupled Semiconductor Lasers," Proc. SPIE 9920 (2016).

[31] Joglekar , Y.N, Wilkey , A. and Vemuri , G., "Lambert Function Methods for Laser Dynamics with

Time-Delayed Feedback," Acta Polytechnica 57, 399-403 (2017).

[32] Feng, L., Wong, Z.J., Ma, R.M., Wang, Y., and Zhang, X., "Single-mode laser by parity-time symmetry breaking," Science 346(6212), 972-975 (2014).

[33] Jing, H., Ozdemir, S.K., Lu, X.Y., Zhang, J., Yang, L., and Nori, F., "PT -symmetric phonon laser," Phys. Rev. Lett. 113, 053604 (2014).

[34] Hodaei, H., Miri, M.A., Heinrich, M., Christodoulides , D.N, and Khajavikhan , M., "Parity- timesymmetric microring lasers," Science 346(6212), 975-978 (2014).

[35] Yidong, C., "Nonlinear optics: asymmetry from symmetry," Nature Physics 10(5), 336-337 (2014).

[36] Feng, L., Xu, Y.L., Fegadolli, W.S., Lu , M.H., Oliviera, J.E.B., Almeida, V.R., Chen , Y.F. and

Scherer, A., "Experimental demonstration of a unidirectional reflectionless parity-time metamaterial at

optical frequencies," Nature Materials 12, 108-113 (2013); Ramezani, H., Kottos, T., Ganainy, R.E. and

Christodoulides , D.N., "Unidirectional nonlinear PT-symmetric optical structures," Phys. Rev. A82, 043803 (2010);

[37] Agarwal, G.S. and Qu, K., "Spontaneous generation of photons in transmission of quantum fields in PT-symmetric optical systems," Phys. Rev. A85, 031802 (R) (2012).

[38] Lang, R. and Kobayashi, K., "External optical feedback effects on semiconductor injection laser properties," IEEE J. Quantum Electronics 16, 347-355 (1980).

[39] Mulet, J., Masoller, C. and Mirasso, C.R., "Modeling bidirectionally coupled single-mode semiconductor lasers," Physical Review A65, 063815 (2002).

[40] Agrawal, G.P. and Dutta, N.K., [Long wavelength semiconductor lasers], Springer, 1986.

[41] Longhi, S., "Phase transitions in wick-rotated pt-symmetry optics," Annals of Physics, 360:150-160 (2015). 